\title{
CHOP/ORP150 Ratio in Endoplasmic Reticulum Stress: A New Mechanism for Diabetic Peripheral Neuropathy
}

\author{
Yuan-Bo Wu ${ }^{a, d} \quad$ Hong-Qi Lib,d Ming-Shan Ren ${ }^{a} \quad$ Wen-Ting Lic $\quad$ Xin-Yi Lva \\ Li Wang $^{a}$
}

aDepartment of Neurology, Provincial Hospital Affiliated to Anhui Medical University, HeFei 230001, China; ${ }^{b}$ Department of Geriatrics, Provincial Hospital Affiliated to Anhui Medical University, HeFei 230001, China; 'Department of Infectious Disease, Provincial Hospital Affiliated to Anhui Medical University, HeFei 230001, China; dAuthors with equal contribution to this study

\section{Key Words}

Diabetic peripheral neuropathy (DPN) - Type II diabetes mellitus - Reactive oxygen species $(\mathrm{ROS}) \cdot$ ER stress $\bullet \mathrm{CHOP} \bullet \mathrm{ORP} 150 \cdot$ Apoptosis $\bullet$ Schwann cells (SCs)

\begin{abstract}
Background/Aims: Peripheral neuropathy is a frequent and severe diabetic complication characterized by progressive loss of peripheral nerve axons and manifested by pain and eventually complete loss of sensation. Effective therapy for diabetic peripheral neuropathy (DPN) is still lacking due to our limited understanding of the mechanisms for nerve injury. Methods: Here we tested the roles of endoplasmic reticulum (ER) stress and the ER stressactivated pro-apoptotic protein CHOP and anti-apoptotic protein ORP150 in DPN in a rat model of high-fat/streptozotocin diabetes and in cultured Schwann cells (SCs). Results: No significant DPN was seen in the early stage of diabetes (4 weeks following verification of diabetes). However, after prolonged diabetes (16 weeks following verification of diabetes), DPN was severely developed as reflected by slowed motor and sensory nerve conduction velocity, blunted thermal nociception, and decreased intraepidermal nerve fiber profiles in the hindpaw. Meanwhile, while it was not noticed in sciatic nerves of early diabetes, ER stress in prolonged diabetic rats was indicated by robust increases in $\mathrm{H}_{2} \mathrm{O}_{2}$ production and expression of the ER chaperon glucose-regulated protein 78 (GRP78). ORP150 expression was substantially upregulated, accompanied by mild increase in $\mathrm{CHOP}$ expression, resulting in a low CHOP/ORP150 ratio, in early diabetes. In contrast, with prolonged diabetes, CHOP expression exceeded ORP150 expression, resulting in an increased CHOP/ORP150 ratio. In vivo knockdown of ORP150 induced DPN in early diabetes and exacerbated the DPN after prolonged diabetes, whereas knockdown of CHOP ameliorated DPN in rats with prolonged diabetic. On the other hand, in vitro knockdown of ORP150 promoted high glucose-induced SC apoptosis, whereas knockdown of CHOP protected SCs from apoptosis. Conclusion: Taken together, we have provided evidence for the critical role of ER stress in the development of DN


and also uncovered CHOP/ORP150 ratio as an important mechanism for determining neuronal apoptosis during ER stress. In the early stage of diabetes, CHOP/ORP150 ratio was relatively low favoring neuronal cell survival, whereas after prolonged diabetes, CHOP/ORP150 ratio increased resulting in apoptotic cell death leading to accelerated DPN.

Copyright $(2013$ S. Karger AG, Basel

\section{Introduction}

A frequent and severe complication of diabetes mellitus is associated with a variety of chronic and acute neuropathies. Diabetic peripheral neuropathies (DPN) are more common and persistent in patients with type 2 than type 1 diabetes [1], affecting each tissue, organ, system and the whole body in approximately $60 \%$ of all patients with the disease, and even portending increased mortality [2]. DPN is a degenerative condition characterized by progressive loss of peripheral nerve axons leading to skin denervation over the duration of diabetes [3-5], predominantly occurring in a distal symmetrical pattern $[5,6]$. DPN is manifested by pain, decreased sensation and eventually complete loss of sensation. Hyperglycemia, the main characteristic of diabetes, produces the microvascular complications of diabetes including neuropathy [7], by causing gross aberrations in global energy balance and metabolism that could lead to the pathophysiological pattern of distal to proximal injury through impaired ability to produce energy at the extremities of long axons far from the cell body. An association between hyperglycemia-derived production of reactive oxygen species (ROS) through the mitochondrial electron transport chain and the development of DPN has been well-recognized $[8,9]$. This association has led to the hypothesis that glycolytic flux is increased in the diabetic peripheral neuronal system $[8,9]$, contributing to mitochondrial ROS generation.

Progress in developing treatments for DPN has been slow due to our limited understanding of how disturbances in metabolic substrates producenerve injury. Vulnerability of neuronal cells to environmental stress contributes to pathogenesis of neurodegenerative disorders. Recently, a growing body of evidence suggested that endoplasmic reticulum (ER) stress plays a key role in the pathogenesis of diabetes mellitus and the associated diabetic peripheral neuropathy as well [10-12]. ER stress is a condition resulting from abnormal folding of newly synthesized proteins in response to a variety of adverse stimulation, particularly production and accumulation of ROS. Upregulation and activation of ER stress proteins is an adaptive mechanism for protein quality control in response to adverse stimuli, but in the face of inducing metabolic disturbances, deregulation of gene expression, and apoptotic cell death after a prolonged period. The apoptotic mechanism is initiated when functions of the ER are severely impaired, to protect the organism by eliminating the damaged cells, with at least three apoptosis pathways being involved in this process [10-12]. The first of these three among the diverse array of proteins induced by ER stress is transcriptional activation of the gene for CCAAT-enhancer-binding protein homologous protein (CHOP) that mediates apoptosis. CHOP is ubiquitously expressed at very low levels [13], but is robustly expressed by perturbations that induce stress in a wide variety of cells. CHOP is present in the cytosol under non-stressed conditions, and stress leads to induction of CHOP and its accumulation in the nucleus [13]. Overexpression of CHOP has been reported to lead to cell cycle arrest and/or apoptosis $[14,15]$, whereas knockout or knockdown of CHOP exhibits reduced apoptosis in response to ER stress [16-19]. On the other hand, oxygen-regulated protein 150 (ORP150) is an essential inducible ER resident heat-shock protein 70 (HSP70) chaperone that is induced by ER stress and hypoxia [20]. ORP150 is upregulated after numerous cellular insults and has a cytoprotective role in renal, neural, and cardiac models of ischemia-reperfusion injury. The cytoprotective effects of ORP150 have also been consistently observed in nerve cells [20-24]. Neurons with increased ORP150 expression demonstrated suppressed caspase-3-like activity. Clearly, ORP150 is an integral participant in cytoprotective pathways during ER stress [24]. Evidently, both CHOP and ORP150 are 
induced during ER stress in neuronal cells, and these two proteins produce opposing effects on apoptosis.

These facts prompted us to ask whether these two proteins play roles in DPN, and if yes, then how do coordinate to decide the fate of neuronal cells? To answer these questions, we carried out the present study in a rate model of type 2 diabetes and a cellular model of ER stress. Our results provide strong evidence for the critical roles of ER stress in the development of DN and also uncover CHOP/ORP150 ratio as a new mechanism for determining neuronal apoptosis during ER stress. In the early stage of ER stress, CHOP/ORP150 ratio was relatively low favoring neuronal cell survival, whereas after prolonged ER stress, CHOP/ORP150 ratio increased resulting in apoptotic cell death leading to accelerated DN.

\section{Materials and Methods}

\section{Rat model of type 2 diabetes}

Male Sprague-Dawley (Slac Laboratory Animal, Shanghai, China) rats 10-11 weeks of age were housed in our animal care facility, and food and water were provided ad libitum. At 12 weeks of age the rats were separated into eight groups: early diabetes (ED; $n=10)$, ED + ORP150-siRNA (ED/siRNA; $n=10), E D+N C$ siRNA (ED/NC siRNA; $n=10)$, prolonged diabetes (PD; $n=10), P D+C H O P-s i R N A(P D / s i R N A ; n=10), P D+N C$ siRNA (PD/NC siRNA; $n=10)$, ED-age-matched control $(n=10)$, and PD-age-matched control $(n=10)$. We used the type 2 diabetes model described by Coppey et al [25]. Briefly, the diabetes groups were fed on a high-fat diet containing $24 \mathrm{gm} \%$ fat, $24 \mathrm{gm} \%$ protein, and $41 \mathrm{gm} \%$ carbohydrate ( $45 \% \mathrm{kcal}$ as fat, $4.7 \mathrm{kcal} / \mathrm{g}$ ) [25]. The primary source of the increased fat content in the diet was lard. The control group was maintained on the control diet ( $3.0 \mathrm{kcal} / \mathrm{g}$ ), which contained $4.25 \mathrm{gm} \%$ fat. Rats were maintained on the high-fat diet for 8 weeks. Afterwards, these rats were treated with streptozotocin $(30 \mathrm{mg} / \mathrm{kg}$ in $0.9 \% \mathrm{NaCl}$. i.p.). Diabetes was verified $96 \mathrm{~h}$ later by evaluating blood glucose levels and rats having blood glucose level of $300 \mathrm{mg} / \mathrm{dL}$ $(11.1 \mathrm{mM})$ or greater were considered to be diabetic. ED rats were maintained on the high-fat diet for an additional 4 weeks prior to experimental measurements; PD rats were maintained on the high-fat diet for an additional 16 weeks and afterwards, they were subject to experimental interventions. All control rats were sham-treated. The use of all animals in this study was approved by, and all operative procedures and animal care strictly conformed to Guidelines set by, the Animal Ethics Committee of the Anhui Medical University.

\section{Glucose tolerance}

Glucose tolerance was determined by i.p. administration of $2 \mathrm{~g} / \mathrm{kg}$ glucose in rats anesthetized with isoflurane, after an overnight fast. Immediately prior to the glucose injection and at 15, 30, 45, 60, 120, 180, and $240 \mathrm{~min}$ blood samples from the tip of the tail were taken to measure circulating glucose levels using glucose oxidase reagent strips with the use of glucose-oxidase reagent strips (Sigma-Aldrich). Hemoglobin A1C levels were determined using a Glyco-tek affinity column kit (Helena Laboratories, Beaumont, TX).

\section{Thermal nociceptive response}

Thermal nociceptive response in the hindpaw was measured using the Hargreaves method as previously described [25,26]. Briefly, the rat was placed in the observation chamber on top of the thermal testing apparatus and allowed to acclimate to the warmed glass surface $\left(30^{\circ} \mathrm{C}\right)$ and surroundings for a period of $15 \mathrm{~min}$. The mobile heat source was adjusted so that it was under the heal of the hindpaw and then activated; this process activates a timer and locally warms the glass surface. When the rat withdrew its paw, the timer and the heat source were turned off and the time was recorded. Three measurements were made for each hindpaw at an interval of $5 \mathrm{~min}$ rest period, and the mean of the three measurements were used as the thermal nociceptive response.

Motor and sensory nerve conduction velocity

On the day of terminal studies rats were weighed and anesthetized with Nembutal i.p. (50 mg/kg, i.p., Sigma-Aldrich). Motor nerve conduction velocity (MNCV) was determined using a noninvasive procedure in the sciatic-posterior tibial conducting system as described elsewhere [25, 26]. The left sciatic nerve was stimulated first at the sciatic notch and then at the Achilles tendon. Stimulation consisted of single 0.2 
Wu et al.: CHOP/ORP150 Ratio and Diabetic Peripheral Neuropathy

ms supramaximal ( $8 \mathrm{~V}$ ) pulses through a bipolar electrode. The evoked potentials were recorded from the interosseous muscle with a unipolar platinum electrode and displayed on a digital storage oscilloscope (JC3202C, Hangzhou Weibo Technology Co., Ltd, China). MNCV was calculated by subtracting the distal from the proximal latency measured in milliseconds (ms) from the stimulus artifact of the take-off of the evoked potential and the difference was divided into the distance between the 2 stimulating electrodes measured in millimeters (mm) using a vernier caliper. Sensory nerve conduction velocity (SNCV) was determined using the digital nerve as described in detail elsewhere [25, 27]. Briefly, hindlimb SNCV was recorded in the digital nerve to the second toe by stimulating with a square-wave pulse of $0.05 \mathrm{~ms}$ duration using the smallest intensity current that resulted in a maximal amplitude response. The sensory nerve action potential (SNAP) was recorded behind the medial malleolus. Sixteen responses were averaged to obtain the position of the negative peak. The maximal SNCV was calculated by measuring the latency to the onset/peak of the initial negative deflection and the distance between stimulating and recording electrodes. The MNCV and SNCV were reported in meters per second.

\section{Intraepidermal nerve fiber density in the hindpaw}

Immunoreactive intraepidermal nerve fiber profiles, which are primarily sensory nerves, were visualized using confocal microscopy. Samples of skin of the right hindpaw were fixed, dehydrated, and embedded in paraffin [25]. Sections $(7 \mu \mathrm{m})$ were collected and immunostained with anti-PGP9.5 antibody (rabbit polyclonal; Abcam, Hong Kong) over night followed by treatment with secondary antibody Alexa Fluor 546 goat anti-rabbit IgG (Invitrogen). Immunoreactive profiles were counted by three individual investigators that were blinded to the sample identity. All profiles within the epidermis were counted and normalized to epidermal length.

\section{Schwann cells (SCS) culture and treatment}

SCs were prepared from sciatic nerves and purified by complement lysis as described by Brockes et al [28]. Cultures were expanded in Dulbecco's modified Eagle's medium (Gibco, Grand Island, NY, USA) supplemented with $10 \%$ (v/v) fetal calf serum. SCs with 30-50\% confluency were transfected with $40 \mathrm{nM}$ siRNA using Lipofectamine ${ }^{\mathrm{TM}}$ RNAiMAX transfection kit (Invitrogen) according to manufacturer's protocols. SCs with $90 \%$ confluency were treated with $200 \mathrm{mM} \mathrm{H}_{2} \mathrm{O}_{2}$ for $24 \mathrm{~h}$. Cells at $48 \mathrm{~h}$ were either collected for gene expression analysis or were treated with ELISA reagent for apoptosis determination.

\section{Small interference RNA (siRNA) treatment}

siRNAs targeting CHOP and ORP150, and a scrambled control siRNA were synthesized by Exiqon. Five nucleotides or deoxynucleotides at both ends of the antisense molecules were locked (the ribose ring is constrained by a methylene bridge between the 2'- $O$ - and the 4'- $C$ atoms) to enhance the cellular stability and target affinity [29]. For in vivo applications, siRNAs were administered to rats through intraperitoneal injection at a dose of $5 \mathrm{mg} / \mathrm{kg} /$ day for consecutive 4 days followed by another three dosages at an interval of 4 days. For in vitro applications, cultured SCs were transfected with the siRNAs using lipofectamine 2000, according to the manufacturer's instructions (Invitrogen). Before siRNA treatment, cells were starved to synchronize growth by incubating in serum- and antibiotics-free medium for $12 \mathrm{~h}$. After $48 \mathrm{hrs}$, cells were collected for experimental measurements.

\section{Enzyme-Linked Immunosorbent Assay (ELISA)}

The Cell Death Detection ELISA kit (Roche Molecular Biochemicals) was employed to quantify DNA fragmentation on the basis of antibody detection of free histone and fragmented DNA, as described elsewhere [30]. SCs Following treatments with $\mathrm{H}_{2} \mathrm{O}_{2}$ and siRNA in 24-well plates $\left(0.7 \times 10^{5}\right.$ cells/well) were incubated in $1 \mathrm{ml}$ of lysis buffer per well for $1 \mathrm{~h}$ at room temperature (RT). The cell lysate was centrifuged at $2000 \mathrm{rpm}$ for $10 \mathrm{~min}$. A supernatant aliquot $(20 \mathrm{ml})$ was added along with $80 \mathrm{ml}$ of the antibody mixture (containing anti-histone and anti-DNA antibodies) to each well of the streptavidin-coated 96-well plate, followed by incubation for $2 \mathrm{~h}$ at RT. The reaction mix was removed, and the substrate solution was added into each well and incubated on a plate shaker at $250 \mathrm{rpm}$ for 5-10 $\mathrm{min}$. The intensity of absorbance at 405 $\mathrm{nm}$ was measured relative to that of substrate solution (blank) with a microplate reader (Rainbow, Tecan), and is expressed as relative optical density (OD) values. 
Assays for caspase 3

Caspase 3 assay kits (Cellular Activity Assay Kit Plus) were purchased from BioMol (Plymouth, PA). After experimental treatment, SCs were centrifuged $\left(1000 \times \mathrm{g}, 4^{\circ} \mathrm{C}, 10 \mathrm{~min}\right)$ and washed with PBS. Cells were resuspended in ice-cold cell lysis buffer and incubated on ice for $10 \mathrm{~min}$. Sample were centrifuged at $10,000 \times \mathrm{g}\left(4^{\circ} \mathrm{C}, 10 \mathrm{~min}\right)$, and the supernatant was removed. Supernatant samples $(10 \mathrm{ml})$ were incubated with $10 \mathrm{ml}$ of substrate $\left(2 \mathrm{mM}\right.$ Ac-DEVDpNA) in $80 \mathrm{ml}$ of assay buffer at $37^{\circ} \mathrm{C}$. Absorbance at $405 \mathrm{~nm}$ was read at several time points until readings reached plateau levels. Optical density changes relative to zerotime values were obtained as a measure of enzyme activity.

\section{Western blot}

Rats were anesthetized by i.p. sodium pentobarbital $(60 \mathrm{mg} / \mathrm{kg})$ and the abdominal cavity was opened. The bilateral sciatic nerves were then quickly removed and frozen in liquid nitrogen [31]. Frozen samples were homogenized in lysis buffer containing $137 \mathrm{mM} \mathrm{NaCl}, 20 \mathrm{mM}$ Tris- $\mathrm{HCl}$ (pH 7.5), 1\% NP40 and a protease inhibitor cocktail (Roche, Penzberg, Germany). The protein concentration was quantified using a BCA Protein Assay Kit (Thermo, IL, USA), and the same amount of total protein was assayed for each immunoblot. Primary antibodies were used at the following dilutions: rabbit polyclonal anti-GRP78, (1:1000, Abcam, MA, USA), anti-CHOP, (1:2500, Santa Cruz, CA, USA), anti-ORP150 (1:2000, Abcam), and anti- $\beta$-actin (1:10000, Sigma, MO, USA). The intensity of immunoreactivity was quantified using Java's freely available Image J software (NIH, MA, USA, http://rsb.info.nih.gov/ij).

\section{Measurement of intracellular ROS}

Oxidative stress was detected by measuring $\mathrm{H}_{2} \mathrm{O}_{2}$ with Amplex Red (Invitrogen), which in the presence of horseradish peroxidase reacts stoichiometrically with $\mathrm{H}_{2} \mathrm{O}_{2}$ to generate the red fluorescent oxidation product resorufin [32,33]. Sciatic nerves (10-mm-long segments proximal to the lesion) were rapidly dissected and homogenized in ice-cold $500 \mathrm{mM}$ phosphate buffer containing $10 \mathrm{mM}$ sodium azide, incubated for $5 \mathrm{~min}$ at room temperature, and centrifuged at $10,000 \times \mathrm{rpm}$ for $10 \mathrm{~min}$ at $4^{\circ} \mathrm{C}$. The supernatant was transferred into a 96-well plate, and samples and $\mathrm{H}_{2} \mathrm{O}_{2}$ standards were incubated with Amplex Red (100 $\mu \mathrm{M})$ and horseradish peroxidase $(0.2 \mathrm{U} / \mathrm{ml})$ for $30 \mathrm{~min}$ at room temperature. Fluorescence intensities were acquired using a plate reader at an excitation of $540 \mathrm{~nm}$ and emission of $595 \mathrm{~nm}$. Experiments were performed in the presence and absence of catalase $(1000 \mathrm{U} / \mathrm{ml})$. Data are presented as the catalase-sensitive part of the Amplex Red oxidation and were normalized to $\mathrm{H}_{2} \mathrm{O}_{2}$ levels in the spinal cord of naive WT mice.

\section{Data analysis}

Results are presented as mean \pm S.E.M. Comparisons between the treatment group and control and nontreated diabetic rats were conducted using one-way ANOVA and Bonferroni after test comparison (SPSS 13.0. statistics software). A $P$ value $<0.05$ was considered significant.

\section{Results}

Characteristics of high-fat/streptozotocin diabetic rats

Data in Table 1 demonstrate that diabetic rats, both the early diabetes group (ED) and the prolonged diabetes group (PD), gained weight similar to their respective agematched control rats. Since there were significant differences in none of the experimental measurements between the two control groups to age-matched ED and PD, respectively, data from these rats were averaged and are presented as one control group in all sections. Mass of the epididymal fat pad was significantly greater in diabetic rats compared to control rats. All diabetic rats were hyperglycemic at the end of the study period as indicated by significantly elevated nonfasting blood glucose and hemoglobin A1C levels (Table 1). Moreover, glucose utilization or tolerance was significantly impaired in diabetic rats (Fig. 1).

\section{Peripheral neuropathy in high-fat/streptozotocin diabetic rats}

Motor and sensory nerve conduction velocity (MNCV and SNCV, respectively), as one of the key indicators of the functional integrity of peripheral nerves, was significantly decreased 
Table 1. Body weight, blood glucose, hemoglobin A1C, and epididymal fat pad in high-fat/streptozotocin diabetic rats. ED: early stage diabetes (4 weeks after verification of diabetes); PD: prolonged diabetes (16 weeks after verification of diabetes); ED siRNA: ORP150 siRNA in the ED group; PD siRNA: CHOP siRNA in the PD group; NC siRNA: scrambled negative control siRNA; HbA1C: hemoglobin A1C.

\begin{tabular}{lcccccccc}
\hline \multicolumn{1}{c}{ Determination } & $\begin{array}{c}\text { Control } \\
\text { for ED }\end{array}$ & $\begin{array}{c}\text { Control } \\
\text { for PD }\end{array}$ & ED & $\begin{array}{c}\text { ED } \\
\text { siRNA }\end{array}$ & $\begin{array}{c}\text { ED } \\
\text { NC siRNA }\end{array}$ & PD & $\begin{array}{c}\text { PD } \\
\text { siRNA }\end{array}$ & $\begin{array}{c}\text { PD } \\
\text { NC siRNA }\end{array}$ \\
\hline $\begin{array}{l}\text { Start weight } \\
\text { g) }\end{array}$ & $308 \pm 7$ & $306 \pm 9$ & $306 \pm 8$ & $311 \pm 11$ & $307 \pm 6$ & $307 \pm 5$ & $310 \pm 7$ & $308 \pm 5$ \\
$\begin{array}{l}\text { End weight } \\
\text { (g) }\end{array}$ & $500 \pm 13$ & $510 \pm 18$ & $496 \pm 10$ & $502 \pm 12$ & $498 \pm 15$ & $492 \pm 16$ & $498 \pm 19$ & $493 \pm 14$ \\
$\begin{array}{l}\text { Blood glucose } \\
\text { (mg/dL) }\end{array}$ & $155 \pm 15$ & $161 \pm 13$ & $365 \pm 21^{*}$ & $341 \pm 33^{*}$ & $360 \pm 19^{*}$ & $383 \pm 28^{*}$ & $375 \pm 22^{*}$ & $385 \pm 28^{*}$ \\
$\begin{array}{l}\text { HbA1C } \\
\text { (\%) }\end{array}$ & $8.3 \pm 0.7$ & $8.6 \pm 0.9$ & $13.5 \pm 1.2^{*}$ & $11.7 \pm 1.7^{*}$ & $13.2 \pm 1.3^{*}$ & $17.7 \pm 2.1^{*}$ & $15.5 \pm 1.8^{*}$ & $16.7 \pm 1.7^{*}$ \\
$\begin{array}{l}\text { Epididymal fat pad } \\
\text { (g) }\end{array}$ & $6.1 \pm 0.4$ & $6.5 \pm 0.5$ & $7.5 \pm 0.7^{*}$ & $7.8 \pm 0.8^{*}$ & $7.6 \pm 0.5^{*}$ & $8.8 \pm 0.9^{*}$ & $8.2 \pm 0.8^{*}$ & $8.5 \pm 0.6^{*}$ \\
\hline
\end{tabular}

Fig. 1. Impairment of glucose utilization or tolerance in both early (ED; 4 weeks after diabetes) and prolonged (PD; 16 weeks after diabetes) diabetic rats. Diabetes was developed by high-fat diet and streptozotocin treatment. Shown are changes of blood glucose concentration as a function of time following glucose injection. ${ }^{* *} p<0.001$ vs control; F-test; $\mathrm{n}=10$ per group.
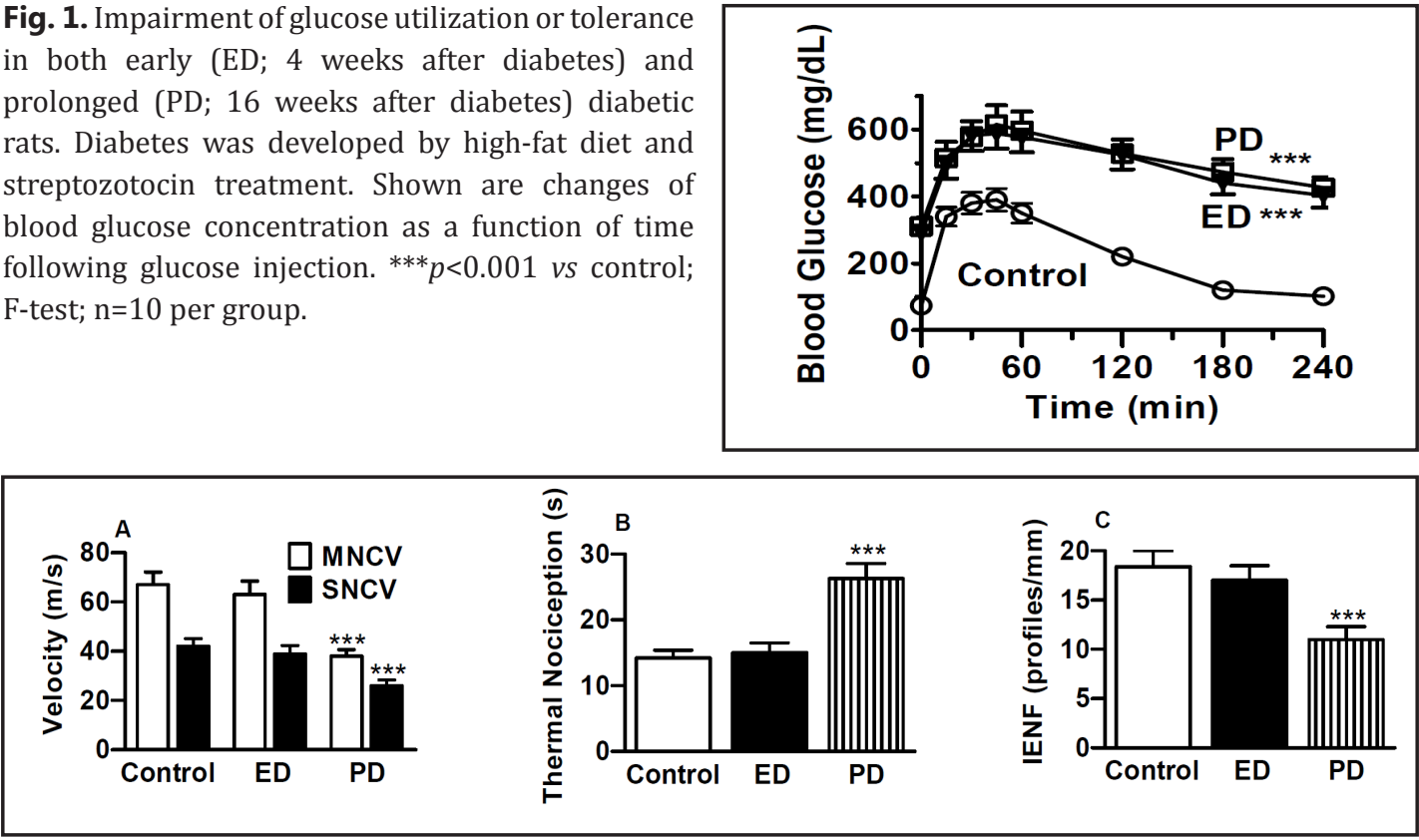

Fig. 2. Diabetic peripheral neuropathy (DPN) revealed by depressed neuronal function in high-fat/ streptozotocin diabetic rats. (A) Slowing of motor and sensory nerve conduction velocity (MNCV and SNCV, respectively) in rats with prolonged diabetes (PD). ${ }^{* * *} p<0.001 v s$ control; t-test; (B) Reduction of thermal nociception in PD rats. ${ }^{* * *} p<0.001$ vs control; t-test; (C) Decrease in intraepidermal nerve fiber (IENF) profiles in the hindpaw of PD rats. Note that PD rats developed the phenotypes of DPN, but ED rats did not. ${ }^{* * *} p<0.001$ vs control; t-test; $\mathrm{n}=10$ per group.

in PDs rats, but not in the ED group, compared to their respective age-matched control animals (Fig. 2A). Moreover, data in Figure 2B demonstrate that PD rats were hypoalgesic to thermal stimuli compared to control rats, whereas ED rats maintained the same level of thermal nociception as control animals. Furthermore, intraepidermal nerve fiber profiles in the hindpaw of PD rats were significantly decreased compared to control rats, but this was not observed in ED animals (Fig. 2C).

\section{ER stress in sciatic nerves of high-fat/streptozotocin diabetic rats}

While the above results clearly indicate the phenotypes of diabetic peripheral neuropathy in the PD model but not in the ED model, it remained unclear what biochemical mechanisms are involved in the pathological process of DPN. Considering the fact that ER 
Fig. 3. Evidence for endoplasmic reticulum (ER) stress in sciatic nerves of high-fat/ streptozotocin diabetic rats. (A) Comparison of $\mathrm{H}_{2} \mathrm{O}_{2}$ production in sciatic nerves, showing an robust increase in $\mathrm{H}_{2} \mathrm{O}_{2}$ production in PD rats, but not in ED rats. ${ }^{* * *} p<0.001 v s$ control; t-test; $n=10$ per group. (B) Upregulation of GRP78 protein level in PD rats, but not in ED rats. ${ }^{* * *} p<0.001$ vs control; t-test; (C) Alterations of CHOP and ORP150 mRNA (left) and protein (right) levels. Note that CHOP and ORP150 were both upregulated; however, the increase in CHOP exceeded that in ORP150 in PD rats, and the opposite was true in ED rats. These changes resulting in an increased CHOP/ORP150 ratio in PD and a decreased CHOP/ORP150 ratio in ED, respectively. ${ }^{*} p<0.05,{ }^{* *} p<0.01$, or ${ }^{* * *} p<0.001$ vs control; t-test.

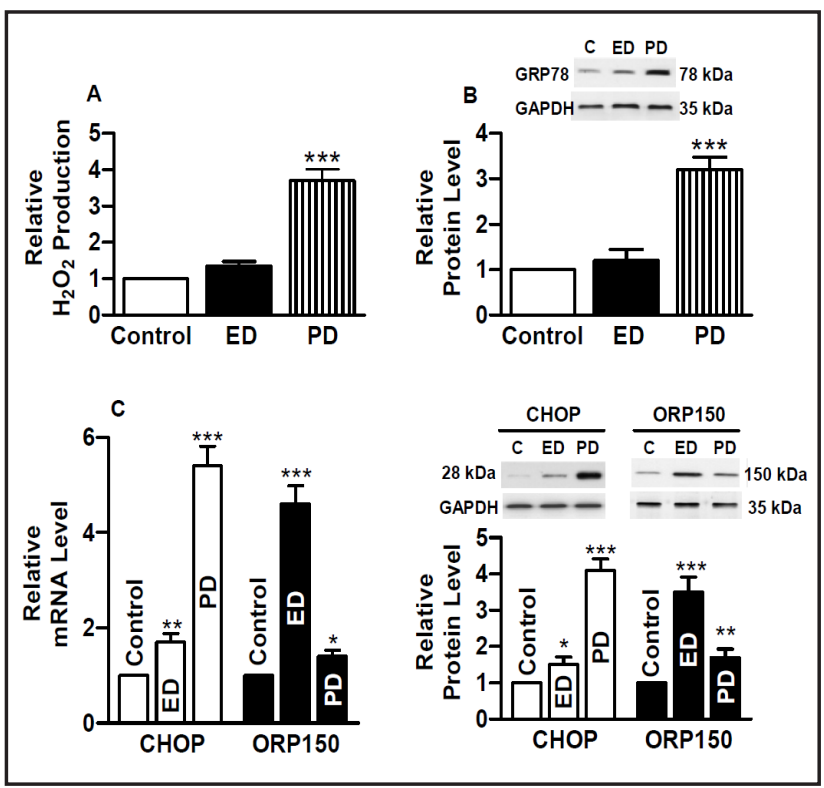

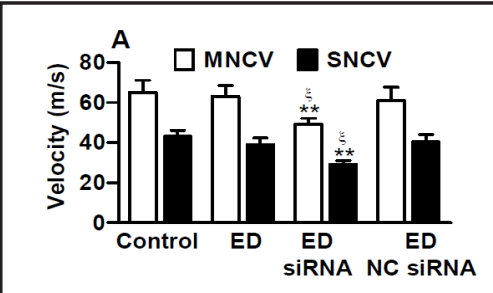
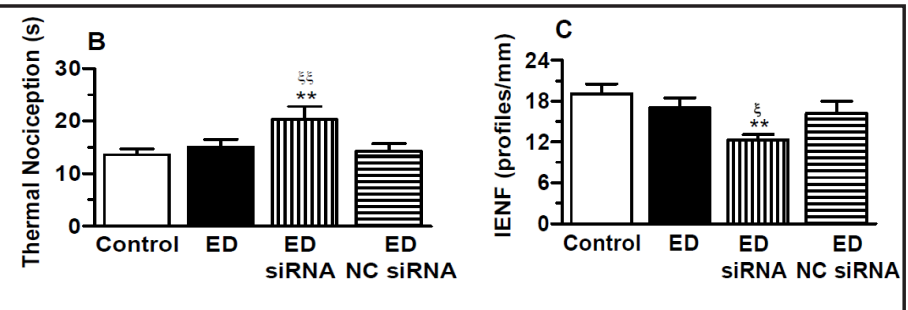

Fig. 4. Effects of knockdown of ORP150 by siRNA on neuronal function in early stage of diabetes (ED) of high-fat/streptozotocin diabetic rats. The siRNA was modified with locked nucleic acid to enhanced cellular stability and target affinity for in vivo applications. It was administered through intraperitoneal injection. Notably, ORP150 siRNA was able to elicit the phenotypes of diabetic peripheral neuropathy (DPN) in ED rats, as reflected by the slowed motor and sensory nerve conduction velocity (MNCV and SNCV, respectively) (A), reduced thermal nociception (B), and decreased intraepidermal nerve fiber (IENF) profiles in the hindpaw (C). ${ }^{* *} p<0.01$ vs control; ${ }^{\xi} p<0.05$ vs ED alone; ${ }^{\xi \xi} p<0.01$ vs ED alone; t-test.

stress plays a critical role in nerve injury, we decided to continue our investigation from this perspective. To this end, we first determined if the redox state in sciatic nerves was adversely altered in diabetes, as oxidative stress is a key factor for ER stress [10-12]. As illustrated in Figure $3 \mathrm{~A}$, the level of basal $\mathrm{H}_{2} \mathrm{O}_{2}$ production in sciatic nerves was relative low in control animals, but it was robustly increased in PD rats and slightly increased in ED rats (Fig. 3A).

The unfolded protein response (UPR) or ER stress is an adaptive survival mechanism in response to the stress of misfolded protein accumulation induced by a wide variety of pathophysiologic and pharmacologic insults. The ER chaperone GRP78 is a central modulator of the UPR [34-36]. We then went on to determine if the GRP78 protein level was affected by diabetes. As depicted in Figure 3B, GRP78 was remarkably upregulated in sciatic nerves of PD rats, relative to control animals. By comparison, GRP78 protein level remained unaltered.

Moreover, CHOP protein level was markedly upregulated (by $4.14 \pm 0.31$ fold), along with a moderate increase in ORP150 protein (by $1.73 \pm 0.23$ fold), resulting in an increased CHOP/ ORP150 ratio, in the PD group (Fig. 3C). In the ED group, however, the increase in ORP150 considerably exceeded that in CHOP $(3.46 \pm 0.42$ for ORP150 vs $1.54 \pm 0.21$ fold for CHOP; Fig. 3C) resulting in a decreased CHOP/ORP150 ratio. The same patterns of changes of CHOP and ORP150 mRNA levels were observed (Fig. 3C, left panel). 

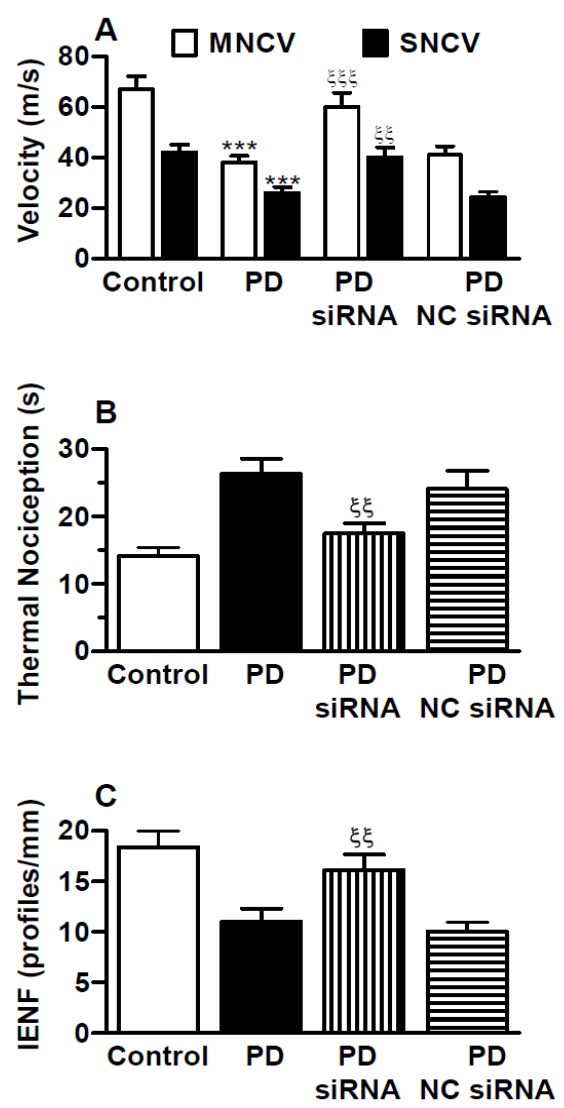

Fig. 5. Effects of knockdown of CHOP by siRNA on neuronal function in prolonged stage of diabetes (PD) of high-fat/streptozotocin diabetic rats. As shown, CHOP siRNA reversed the phenotypes of diabetic peripheral neuropathy (DPN) in PD rats, as reflected by the recovery of slowed MNCV and SNCV (A), reduced thermal nociception (B), and decreased intraepidermal nerve fiber (IENF) profiles in the hindpaw (C). ${ }^{* * *} p<0.001$ vs control; ${ }^{\xi \xi} p<0.01$ vs ED alone; ${ }^{\xi \xi \xi} p<0.001$ vs ED alone; t-test.

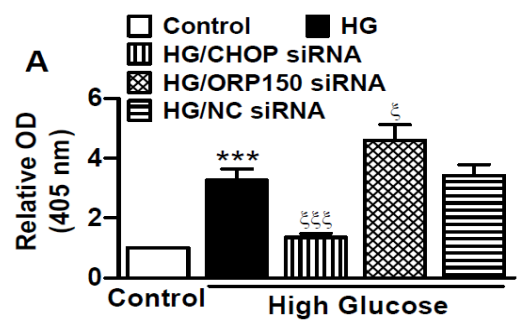

B

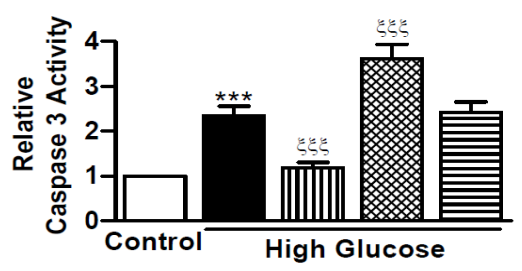

C
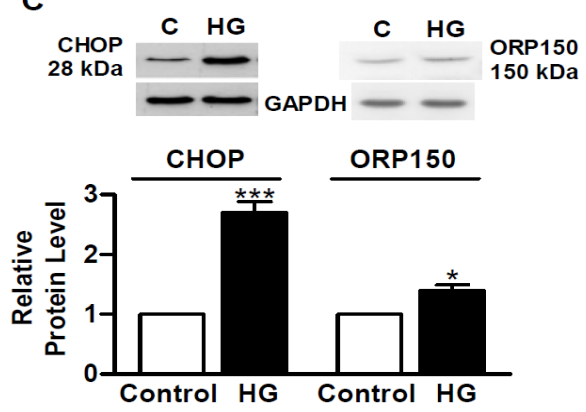

Fig. 6. Effects of knockdown of CHOP or ORP150 by siRNA on high glucose-induced apoptosis in Schwann cells (SCs) of rats. (A) Apoptosis of SCs reported by DNA fragmentation determined by ELISA and expressed as relative optical density (OD at 405 $\mathrm{nm}$ ); (B) Apoptosis reported by increased caspase 3 activity, the executioner of apoptosis; (C) Expression of both CHOP and ORP150 proteins was upregulated in SCs treated by high glucose, but the magnitude of upregulation was more prominent for CHOP than for ORP150, resulting in a CHOP/ORP150 ratio $>1$. ${ }^{* * *} p<0.001 \mathrm{HG}$ vs control; ${ }^{\xi \xi} p<0.01$ vs ED alone; ${ }^{\xi \xi \xi} p<0.001$ vs ED alone; t-test.

Knockdown of ORP150 exacerbates DPN in high-fat/streptozotocin diabetic rats

ORP150 has been documented to exert protective effects on neurons [20-23]. The observed upregulation of ORP150 in ED rats is expected to contribute to the retardation of development of DPN; conversely, knockdown of ORP150 should evoke DPN phenotypes in the setting of ED. To test this notion, we applied the loss-of-function of ORP150 to rats of the ED group using ORP150 siRNA with locked nucleic acid. As shown in Figure 4, moderate by significant DPN was elicited in ED rats, as indicated by decelerated MNCV and SNCV (Fig. 4A), blunted thermal nociception (Fig. 4B), and reduced intraepidermal nerve fiber profiles in the hindpaw (Fig. 4C). The scrambled siRNA as a negative control construct (NC siRNA) failed to affect these nerve functions (Fig. 4). 
Fig. 7. Verification of the efficiency and specificity of siRNAs to knockdown CHOP or ORP150 in vivo in sciatic nerves of $\mathrm{ED}$ or $\mathrm{PD}$ rats $(\mathrm{A}$ $\& \mathrm{C}$ ) and in vitro in SCs (B \& D). Note (1) the lack of effect of NC siRNA (scrambled negative control construct) on CHOP and ORP150, (2) the lack of effects of CHOP siRNA on ORF150 and GRP78, and (3) the lack of effects of ORF150 siRNA on CHOP and GRP78. ${ }^{* * *} p<0.001$ siRNA vs NC siRNA; t-test.
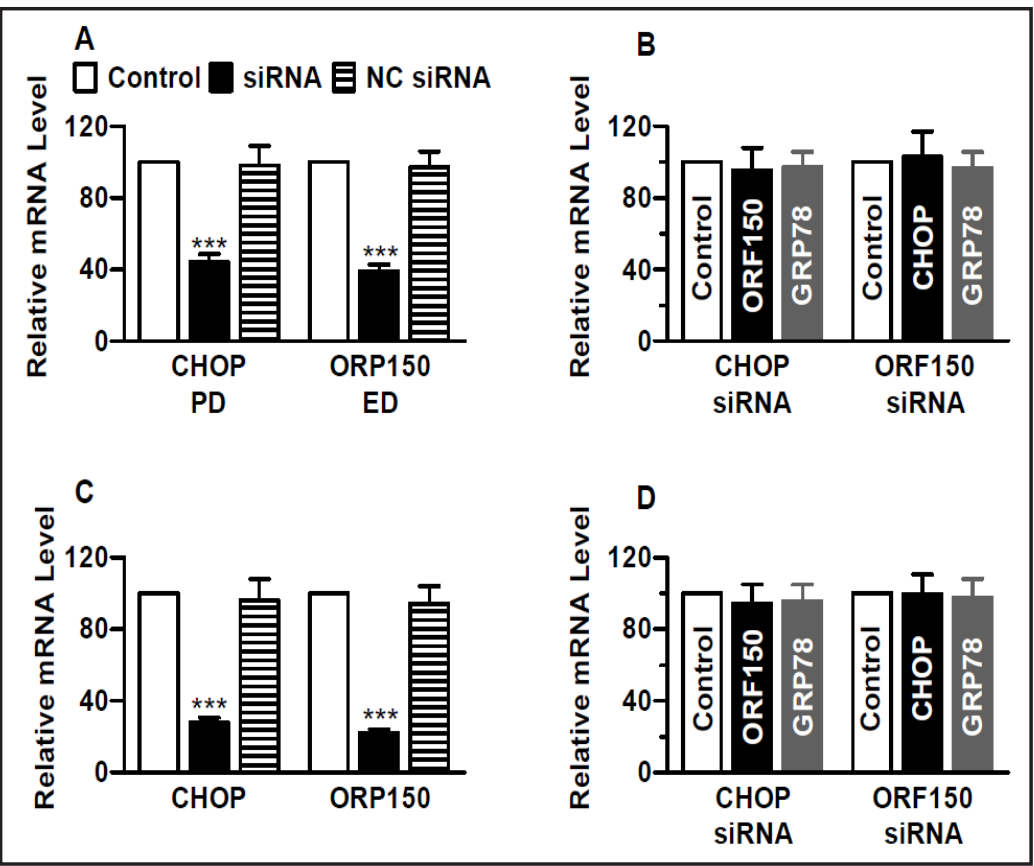

Knockdown of CHOP ameliorates DPN in high-fat/streptozotocin diabetic rats

A notable finding in this study was robust upregulation of CHOP protein expression (Fig. 3C). This protein has been shown to produce pro-apoptotic effects during ER stress [14-18]. Upregulation of CHOP in PD rats may thus account for, at least partially, the DPN in our model. If it is true, then knockdown of CHOP should be able to reverse DPN. Our results shown in Figure 5 indeed provide strong evidence for this notion: in vivo knockdown of CHOP by siRNA alleviated the phenotypes of DPN in PD rats. CHOP siRNA accelerated the depressed MNCV and SNCV (Fig. 5A), sharpen the blunted thermal nociception (Fig. 5B), and preserved the intraepidermal nerve fiber profiles in the hindpaw (Fig. 5C). The NC siRNA altered one of these parameters (Fig. 5).

\section{Effects of CHOP and ORP150 siRNAs on apoptosis of Schwann cells}

In response to peripheral nerve injury, myelinating Schwann cells are activated and their myelin properties are modified, resulting in altered conduction properties of nociceptive fibers [37-40]. Apoptosis of SCs plays a primary role in the development of DPN [41, 42]. To see if how CHOP and ORP150 affect SC apoptosis in the setting of diabetes, we turned to investigate the effects of siRNAs to these molecules in the presence of high glucose to simulate hyperglycemia. Data in Figure 6 demonstrate remarkable apoptosis in SCs incubated with high glucose, as reflected by increased DNA fragmentation (Fig. 6A) and enhanced caspase 3 activity (Fig. 6B) in these cells. Treatment of SCs with CHOP siRNA rescued the cells from apoptosis, whereas treatment with ORP150 siRNA exacerbated apoptotic cell death (Fig. 6A \& 6B). NC siRNA did not produce any of these changes (Fig. 6A \& 6B). Consistently, CHOP protein level was pronouncedly increased along with only moderate elevation of ORP150 (Fig. 6C).

Finally, we verified the ability of the CHOP siRNA and ORP150 siRNA to knockdown their respective genes in vivo in sciatic nerves of ED or PD rats (Fig. 7A) and in vitro in SCs (Fig. 7B). We also obtained evidence for the specificity of the siRNAs used in our study: (1) The NC siRNA had no significant effects on CHOP and ORP150 expression; (2) CHOP siRNA failed to affect ORF150 and GRP78 transcripts in vivo (Fig. 7B) and in vitro (Fig. 7D); and (3) ORF150 siRNA did not alter mRNA levels of CHOP and GRP78 (Fig. 7B \& 7D). 


\section{Discussion}

\section{Main findings}

Diabetic peripheral neuropathy (DPN) is one of the most frequently encountered complications and is characterized by progressive loss of peripheral nerve axons and manifested by pain and eventually complete loss of sensation. Effective therapy for DPN is still lacking due to our limited understanding of this problem. Here, by employing a rat model of type 2 diabetes of high-fat/streptozotocin combination and a cellular model of simulated hyperglycemia with Schwann cells (SCs), we tested the roles of endoplasmic reticulum (ER) stress and the ER stress-activated pro-apoptotic protein CHOP and anti-apoptotic protein ORP150 in DPN. The main findings in this study include: (1) No DPN was developed in the early stage of diabetes (4 weeks after diabetes), but severe DPN was consistently attained after prolonged period of diabetes (16 weeks of diabetes). The phenotypes of DPN included slowed motor and sensory nerve conduction velocity, impaired thermal nociception, and decreased intraepidermal nerve fiber profiles in the hindpaw; (2) Coincidently, at the subcellular level, while ER stress was not noticed in sciatic nerves of early diabetes, it was prominent in prolonged diabetes being manifested by robust increases in $\mathrm{H}_{2} \mathrm{O}_{2}$ production and expression of the ER chaperon glucose-regulated protein 78 (GRP78); (3) ORP150 expression was substantially upregulated, accompanied by mild increase in CHOP expression in early diabetes, with a low CHOP/ORP150 ratio. In contrast, with prolonged diabetes, CHOP expression exceeded ORP150 expression resulting in a substantially increased CHOP/ ORP150 ratio; (4) In vivo knockdown of ORP150 to increase CHOP/ORP150 ratio rendered development of DPN in early diabetes. In contrast, knockdown of CHOP to decrease CHOP/ ORP150 ratio ameliorated DPN in rats with prolonged diabetic; (5) On the other hand, in vitro knockdown of ORP150 promoted high glucose-induced SC apoptosis, whereas knockdown of CHOP protected SCs from apoptosis. Taken together, we have provided evidence for the critical role of ER stress in the development of DN and also uncovered CHOP/ORP150 ratio as an important mechanism for determining neuronal apoptosis during ER stress. In the early stage of diabetes, CHOP/ORP150 ratio was relatively low favoring neuronal cell survival, whereas after prolonged diabetes, CHOP/ORP150 ratio increased resulting in apoptotic cell death leading to accelerated DPN.

\section{Comparison with previous studies}

The high fat fed/low dose streptozotocin diabetic rats are an animal model for type 2 diabetes [43, 44]. According to Coppey et al. [25] and Reed et al. [43], the diabetes in these rats is analogous to the development of human type 2 diabetes when the decline in hyperinsulinemia is not able to compensate for insulin resistance and hyperglycemia occurs. In our hands, the 4-week rat models early stage, whereas the 16-week rat models late stage, type 2 diabetes [45]. Coincidently, the rats of early diabetes did not develop notable ER stress and DPN, while those of late diabetes were characterized with severe ER stress and DPN.

Schwann cells are the principal glia of the peripheral nervous system, which function to support neurons. Schwann cells wrap around axons of motor and sensory neurons to form the myelin sheath. They are involved in many important aspects of peripheral nerve biologythe conduction of nervous impulses along axons, nerve development and regeneration, trophic support for neurons, production of the nerve extracellular matrix, trophic support for neurons, production of the nerve extracellular matrix, modulation of neuromuscular synaptic activity, maintenance of axons and are crucial for neuronal survival, etc. The phenotypic alterations of peripheral nerve function in late stage diabetes in this study may well be ascribed to Schwann cell injury and dysfunction. Schwann cell injury is expected to slow motor and sensory nerve conduction velocity, impair thermal nociception, and decrease intraepidermal nerve fiber profiles [37-40]. Moreover, the apoptosis of Schwann cells induced by hyperglycemic insult may account at least partially for the progressive loss of peripheral nerve axons leading to skin denervation $[41,42]$. 
One mechanism that could explain the observed apoptosis of Schwann cells and reduced intraepidermal nerve fiber profiles is the development of ER stress in our models. ER stress is an important biochemical mechanism for protein quality control and cell death as well $[10-12,46]$. A number of signalling pathways are activated during ER stress, which either mediate survival or apoptotic signal. The balance between the pro-apoptotic and anti-apoptotic forces determines the cell fate under the conditions of ER stress. CHOP is among the ER stress-induced pro-apoptotic factor. Our results demonstrate that though CHOP expression was upregulated in both early and late diabetes, the upregulation in late diabetes was far stronger than in early diabetes. In contrast, the changes of ORP150 expression showed an opposite pattern: the magnitude of ORP150 upregulation was much greater in early than in late diabetes. Consistent with previous studies [16-24], our results demonstrate that CHOP elicited damaging effects whilst ORP150 produced cytoprotective actions on Schwann cells and sciatic nerves. Moreover, in late diabetes, the delayed but robust increase in CHOP expression overweighed the mild increase in ORP150 expression, resulting in manifestation of the adverse effects of ER stress leading to DPN. In agreement with this notion, knockdown of CHOP alleviated the phenotypes of DPN in vivo and apoptosis in Schwann cells in vitro. In early diabetes, however, the early increase in CHOP expression was mild compared to the robust increase in ORP150 expression and the adverse effects of CHOP might be offset by the beneficial actions of ORP150; thus, no DPN was detected. Most strikingly, is the observation that . These data suggest that the ratio of CHOP to ORP150 is more important than the individual level of these two proteins in determining the cell fate and the associated function of peripheral nerves. However, we admit that more detailed experiments are required to verify this issue.

\section{Potential implications of the study}

The findings of this study imply that DPN develops only with prolonged diabetic status when severe ER stress has been developed; therefore interventions can well be implemented during this lag period to retard or even prevent DPN. One of the possible interventions is to knockdown CHOP expression, based on our results showing the aberrant upregulation of CHOP and the efficacy of CHOP siRNA in ameliorating DPN. Alternatively, overexpression of ORP150 should also be able to achieve the same goal of alleviating DPN. The principle is to reduce the ratio of CHOP/ORP150. Though our data are not conclusive, but open up an opportunity for future studies on this sissue.

\section{Possible limitations of the study}

Precaution must be taken when interpreting our data from systemic administration of siRNAs, as the improvement of peripheral nerve function or relief of DPN may result from indirect effects on other organs/tissues. For instance, the siRNAs may act on pancreatic $\beta$-cells or on the metabolic pathways to alter the diabetic status that in turn changes DPN, albeit our data summarized in Table 1 indicate that the siRNAs did not affect blood glucose and HbA1C concentrations. Although it appears that CHOP/ORP150 ratio plays a more important role in DPN than either of these two proteins, no reliable and definite values on the ratio could be obtained using Western blot analysis, as different antibodies are not comparable in quantification.

\section{Conclusions}

Collectively, we have presented evidence for the involvement of ER stress in the development of DPN in the setting of type 2 diabetes. In particular, the signaling mediators of ER stress CHOP and ORP150 play important and counteracting parts in determining the development of DPN, with CHOP favoring DPN and ORP150 being against DPN. 
Wu et al.: CHOP/ORP150 Ratio and Diabetic Peripheral Neuropathy

\section{Conflicts of Interest}

The authors declare no conflicts of interest for this study.

\section{References}

1 Young MJ, Boulton AJ, MacLeod AF, Williams DR, Sonksen PH: A multicentre study of the prevalence of diabetic peripheral neuropathy in the United Kingdom hospital clinic population. Diabetologia 1993;36:150-154.

2 Vincent AM, Feldman EL: New insights into the mechanisms of diabetic neuropathy. Rev Endocr Metab Disord 2004;5:227-236.

3 Johnson PC, Doll SC, Cromey DW: Pathogenesis of diabetic neuropathy. Annals of Neurology 1986;19:450457.

-4 Shun CT, Chang YC, Wu HP, Hsieh SC, Lin WM, Lin YH, Tai TY, Hsieh ST: Skin denervation in type 2 diabetes: correlations with diabetic duration and functional impairments. Brain 2004;127:1593-1605.

5 Said G: Diabetic neuropathy--a review. Nat Clin Pract Neurol 2007;3:331-340.

-6 Sullivan KA, Hayes JM, Wiggin TD, Backus C, Su Oh S, Lentz SI, Brosius F 3rd, Feldman EL: Mouse models of diabetic neuropathy. Neurobiol Dis 2007;28:276-285.

7 Chisholm DJ: The Diabetes Control and Complications Trial (DCCT). A milestone in diabetes management. Med J Aust 1993;159:721-723.

8 Brownlee M: Biochemistry and molecular cell biology of diabetic complications. Nature 2001;414:813820 .

-9 Tomlinson DR, Gardiner NJ: Glucose neurotoxicity. Nat Rev Neurosci 2008;9:36-45.

10 Lupachyk S, Watcho P, Stavniichuk R, Shevalye H, Obrosova IG: Endoplasmic reticulum stress plays a key role in the pathogenesis of diabetic peripheral neuropathy. Diabetes 2013;62:944-52.

11 Cameron NE: Role of endoplasmic reticulum stress in diabetic neuropathy. Diabetes 2013;62:696-697.

12 Li L, Ye XP, Lu AZ, Zhou SQ, Liu H, Liu ZJ, Jiang S, Xu SY: Hyperglycemia magnifies bupivacaine-induced cell apoptosis triggered by mitochondria dysfunction and endoplasmic reticulum stress. J Neurosci Res 2013;91:786-798.

13 Ron D, Habener JF: CHOP, a novel developmentally regulated nuclear protein that dimerizes with transcription factors C/EBP and LAP and functions as a dominant-negative inhibitor of gene transcription. Genes Dev 1992;6:439-453.

14 Barone MV, Crozat A, Tabaee A, Philipson L, Ron D: CHOP (GADD153) and its oncogenic variant, TLS-CHOP, have opposing effects on the induction of G1/S arrest. Genes Dev 1994;8:453-464.

15 Gotoh T, Oyadomari S Mori K, Mori M: Nitric oxide-induced apoptosis in RAW 264.7 macrophages is mediated by endoplasmic reticulum stress pathway involving ATF6 and CHOP. J Biol Chem 2002;277:12343-12350.

- 16 Zinszner H, Kuroda M, Wang X, Batchvarova N, Lightfoot RT, Remotti H, Stevens JL, Ron D: CHOP is implicated in programmed cell death in response to impaired function of the endoplasmic reticulum. Genes Dev 1998;12:982-995.

17 Oyadomari S, Koizumi A, Takeda K, Gotoh T, Akira S, Araki E, Mori M: Targeted disruption of the Chop gene delays endoplasmic reticulum stress-mediated diabetes. J Clin Invest 2002;109:525-532.

18 Oyadomari S, Mori M: Roles of CHOP/GADD153 in endoplasmic reticulum stress. Cell Death Differ 2004;11:381-389.

19 Gow A, Wrabetz L: CHOP and the endoplasmic reticulum stress response in myelinating glia. Curr Opin Neurobiol. 2009;19:505-510.

-20 Kitao Y, Ozawa K, Miyazaki M, Tamatani M, Kobayashi T, Yanagi H, Okabe M, Ikawa M, Yamashima T, Stern DM, Hori O, Ogawa S: Expression of the endoplasmic reticulum molecular chaperone (ORP150) rescues hippocampal neurons from glutamate toxicity. J Clin Invest 2001;108:1439-1450.

21 Tamatani M, Matsuyama T, Yamaguchi A, Mitsuda N, Tsukamoto Y, Taniguchi M, Che YH, Ozawa K, Hori O, Nishimura H, Yamashita A, Okabe M, Yanagi H, Stern DM, Ogawa S, Tohyama M: ORP150 protects against hypoxia/ischemia-induced neuronal death. Nat Med 2001;7:317-323.

22 Miyazaki M, Ozawa K, Hori O, Kitao Y, Matsushita K, Ogawa S, Matsuyama T: Expression of 150-kd oxygenregulated protein in the hippocampus suppresses delayed neuronal cell death. J Cereb Blood Flow Metab 2002;22:979-987. 
Wu et al.: CHOP/ORP150 Ratio and Diabetic Peripheral Neuropathy

23 Kitano H, Nishimura H, Tachibana H, Yoshikawa H, Matsuyama T: ORP150 ameliorates ischemia/ reperfusion injury from middle cerebral artery occlusion in mouse brain. Brain Res 2004;1015:122-128.

24 Kusaczuk M, Cechowska-Pasko M: Molecular Chaperone ORP150 in ER Stress-related Diseases. Curr Pharm Des 2013;19:2807-2818.

-25 Coppey LJ, Holmes A, Davidson EP, Yorek MA: Partial replacement with menhaden oil improves peripheral neuropathy in high-fat-fed low-dose streptozotocin type 2 diabetic rat. J Nutr Metab 2012;2012:950517.

26 Oltman CL, Davidson EP, Coppey LJ, Kleinschmidt TL, Lund DD, Yorek MA: Attenuation of Vascular/Neural Dysfunction in Zucker Rats Treated with Enalapril or Rosuvastatin. Obesity 2008;16:82-89.

27 Obrosova IG, Li F, Abatan OI, Forsell MA, Komjati K, Pacher P, Szabo C, Stevens MJ: Role of poly(ADP-ribose) polymerase activation in diabetic neuropathy. Diabetes 2004;53:711-720.

28 Brockes JP, Fields KL, Raff MC: Studies on cultured rat Schwann cells. I. Establishment of purified populations from cultures of peripheral nerve. Brain Res 1979;165:105-118.

-29 Elmén J, Thonberg H, Ljungberg K, Frieden M, Westergaard M, Xu Y, Wahren B, Liang Z, Ørum H, Koch T, Wahlestedt C: Locked nucleic acid (LNA) mediated improvements in siRNA stability and functionality. Nucleic Acids Res 2005;33:439-447.

30 Han H, Wang H, Long H, Nattel S, Wang Z: Oxidative preconditioning and apoptosis in L-cells. Roles of protein kinase B and mitogen-activated protein kinases. J Biol Chem 2001;276:26357-26364.

- 31 Zhou J-Y, Zhou S-W: Protection of trigonelline on experimental diabetic peripheral neuropathy. Evid Based Complement Alternat Med 2012;2012:164219.

-32 Kallenborn-Gerhardt W, Schröder K, Del Turco D, Lu R, Kynast K, Kosowski J, Niederberger E, Shah AM, Brandes RP, Geisslinger G, Schmidtko A: NADPH oxidase-4 maintains neuropathic pain after peripheral nerve injury. J Neurosci 2012;32:10136-10145.

-33 Rhee SG, Chang TS, Jeong W, Kang D: Methods for detection and measurement of hydrogen peroxide inside and outside of cells. Mol Cells 2010;29:539-549.

-34 Ni M, Lee AS: ER chaperones in mammalian development and human diseases. FEBS Lett 2007;581:36413651.

-35 Katayama T, Imaizumi K, Manabe T, Hitomi J, Kudo T, Tohyama M: Induction of neuronal death by ER stress in Alzheimer's disease. J Chem Neuroanat 2004;28:67-78.

-36 Kim R, Emi M, Tanabe K, Murakami S: Role of the unfolded protein response in cell death. Apoptosis 2006;11:5-13.

37 Shin YK, Jang SY, Lee HK, Jung J, Suh DJ, Seo SY, Park HT: Pathological adaptive responses of Schwann cells to endoplasmic reticulum stress in bortezomib-inducedperipheral neuropathy. Glia 2010;58:1961-1976.

-38 Thacker MA, Clark AK, Marchand F, McMahon SB: Pathophysiology of peripheral neuropathic pain: immune cells and molecules. Anesth Analg 2007;105:838-847.

-39 Nagai J, Uchida H, Matsushita Y, Yano R, Ueda M, Niwa M, Aoki J, Chun J, Ueda H: Autotaxin and lysophosphatidic acid1 receptor-mediated demyelination of dorsal root fibers by sciatic nerve injury and intrathecal lysophosphatidylcholine. Mol Pain 2010;6:78. doi: 10.1186/1744-8069-6-78.

40 Devor M: Centralization, central sensitization and neuropathic pain. Focus on "sciatic chronic constrictioninjury produces cell-type-specific changes in the electrophysiological properties of rat substantia gelatinosa neurons". J Neurophysiol 2006;96:522-523.

41 Askwith T, Zeng W, Eggo MC, Stevens MJ: Taurine reduces nitrosative stress and nitric oxide synthase expression in high glucose-exposed human Schwann cells. Exp Neurol 2012;233:154-162.

42 Vincent AM, Callaghan BC, Smith AL, Feldman EL: Diabetic neuropathy: cellular mechanisms as therapeutic targets. Nat Rev Neurol 2011;7:573-583.

43 Reed MJ, Meszaros K, Entes LJ, Claypool MD, Pinkett JG, Gadbois TM, Reaven GM: A new rat model of type 2 diabetes: the fat-fed, streptozotocin-treated rat. Metabolism 2000;49:1390-1394.

-44 Srinivasan K, Viswanad B, Asrat L, Kaul CL, Ramarao P: Combination of high-fat diet-fed and low-dose streptozotocin-treated rat: a model for type 2 diabetes and pharmacological screening. Pharmacol Res 2005;52:313-320.

45 Davidson EP, Coppey LJ, Holmes A, Dake B, Yorek MA: Effect of treatment of high fat fed/low dose streptozotocin-diabetic rats with Ilepatril on vascular and neural complications. European J Pharmacol 2011;688:497-506.

46 Tabas I, Ron D: Integrating the mechanisms of apoptosis induced by endoplasmic reticulum stress. Nat Cell Biol 2011;13:184-190. 